\title{
Stable Manifolds and the Transition to Turbulence in Pipe Flow
}

\author{
By ${ }^{1}$ D. VISWA NATH AND ${ }^{2}$ P. CVITANOVIĆ \\ ${ }^{1}$ Department of Mathematics, University of Michigan, Ann Arbor, MI 48109, USA \\ ${ }^{2}$ School of Physics, Georgia Institute of Technology, Atlanta, GA 30332, USA
}

(Printed 29 October 2018)

Lower-branch traveling waves and equilibria computed in pipe flow and other shear flows appear intermediate between turbulent and laminar motions. We take a step towards connecting these lower-branch solutions to transition by deriving a numerical method for finding certain special disturbances of the laminar flow in a short pipe. These special disturbances cause the disturbed velocity field to approach the lower-branch solution by evolving along its stable manifold. If the disturbance were slightly smaller, the flow would relaminarize, and if slightly larger, it would transition to a turbulent state.

\section{Introduction}

The connection between the law of resistance to water flowing in a tube and the sinuous or direct nature of the internal motion of the fluid was established by Reynolds (1883). In the transitional regime, he observed "flashes" and recorded them in Figure 16 of his paper. Later experiments using hot-wire measurements have revealed the structure of puffs and slugs (Wygnanski and Champagne 1973). Particularly intriguing are equilibrium puffs that maintain their spatial extent as they travel downstream with a characteristic speed (Wygnanski et al. 1975). Such puffs are approximately 20 pipe diameters long and are observed for Re (Reynolds number) somewhat greater than 2000. Further, the structure of the puff is independent of the disturbance used to create it.

For a range of $R e$, the flow injected into the pipe assumes the familiar Hagen-Poiseuille laminar profile downstream. The flow does not have the laminar profile at the inlet. Therefore it is important to distinguish between disturbances at the inlet and disturbances to fully developed laminar flow (Willis et al. 2008)[Willis, Peixinho et al.]. The early experiments used inlet disturbances, but in theoretical investigations such as this one, it has been common practice to consider disturbances to the laminar flow.

In their experiments to determine the dependence of the threshold for transition on Re, Darbyshire and Mullin (1995) used a constant mass-flux pipe and introduced disturbances to the laminar flow at a point sufficiently downstream from the inlet. They were able to determine thresholds over a range of $R e$, but Figure 11 and other figures in their paper showed that certain disturbances above the threshold do not transition while certain disturbances below the threshold do transition. Hof et al. (2003) determined that the threshold scaled as $R e^{\alpha}$ with $\alpha=-1$, when the laminar flow was disturbed by a single boxcar pulse of fluid injected at six different points. Mellibovsky and Meseguer (2007) have reproduced $\alpha=-1$ in a numerical study that added a body force term to the Navier-Stokes equation to model the effect of the boxcar pulse of fluid. For different disturbances of the laminar flow, Peixinho and Mullin (2007) found $\alpha<-1$. In that experiment, the transition is sequential, with flow visualizations showing the disturbance 
changing its form as it travels downstream before leading to bigger structures. Following O'Sullivan and Breuer (1994), Peixinho and Mullin (2007) point out that disturbances that lead to $\alpha<-1$ probably do not significantly distort the mean flow.

With regard to theory, Reynolds's assertion that "there was small chance of discovering anything new or faulty" in the Navier-Stokes equation has stood the test of time. Thanks to numerical computations, we now know that the incompressible Navier-Stokes equation adequately explains a remarkable wealth of phenomena related to transitional turbulence and fully developed turbulence. Although the Navier-Stokes equation can be solved numerically in certain regimes, the nature of the solutions of that equation has proved difficult to understand.

It is clear, however, that the nature of the solutions is quite different in the turbulent and transitional regimes. Fully developed turbulence is characterized by rapid decay of correlations and fine scales. Statistical theories that separate turbulent velocity fields into means and fluctuations have had significant successes (Narasimha 1989), even though coherent motions are present in certain regions of fully developed turbulence (Robinson 1991). In contrast, the transition problem seems to be fundamentally dynamical in nature.

Following the experiments of Darbyshire and Mullin (1995), Faisst and Eckhardt (2004) and Schmiegel and Eckhardt (1997) argued that there is no sharp boundary between initial conditions that trigger turbulence and those that do not, and demonstrated computationally that the stability border for plane Couette flow is a fractal. One of their suggestions, namely that a chaotic saddle could be present for transitional Re, illustrates the dynamical nature of the transition problem.

More recently, Faisst and Eckhardt (2003) and Wedin and Kerswell (2004) computed a number of traveling wave solutions of pipe flow. Their work was preceded by computations of somewhat similar solutions of channel flows by Nagata (1990) and Waleffe (1998). Hof et al. (2004) found streak patterns in puffs and slugs that appeared close to those of some pipe flow traveling waves. The traveling waves were computed in short pipes, typically only a few pipe diameters long, while puffs are as long as 20 pipe diameters. Therefore the following question may be asked: do the experimentally observed structures correspond to the computed traveling waves?

The correlation functions, such as those of Schneider et al. (2007a), used to detect streak patterns in experimental or numerical flow fields look for $m$-fold rotational symmetry with respect to the pipe axis. Using such a correlation function, Figure 5 of Schneider et al. (2007a) illustrates a transition from a four streak state to a six streak state within a spatial range of a single pipe radius. Willis and Kerswell (2008) found structures with $m=3$ and $m=4$ within streamwise distances of about 2 pipe diameter preceding the trailing edge of the puff and 5 pipe diameters following the trailing edge. Figure 5 of their paper gives some evidence that parts of the puff on either side of its trailing edge (but not at the trailing edge itself) visit traveling wave solutions with $m=3$ and $m=4$. It could be significant that Figures 7 and 23 of Wedin and Kerswell (2004) (which use different units) imply that some of the $m=3$ and $m=4$ traveling waves have wave speeds relatively close to that of the puff. Willis and Kerswell (2008) also found that the qualitative comparisons of (Hof et al. 2004) which used slug cross-sections had significant problems. Instead of the trailing edge of the puff, Eckhardt and Schneider (2008) used the center of turbulent energy to fix a position within a moving puff. Their center of turbulent energy is more precisely defined and it moves with the puff in a quite regular manner. They found the axial correlation lengths around that center to be quite short. While the question raised in the previous paragraph cannot be answered conclusively at the moment, these arguments suggest that short pipe computations are of some relevance. 

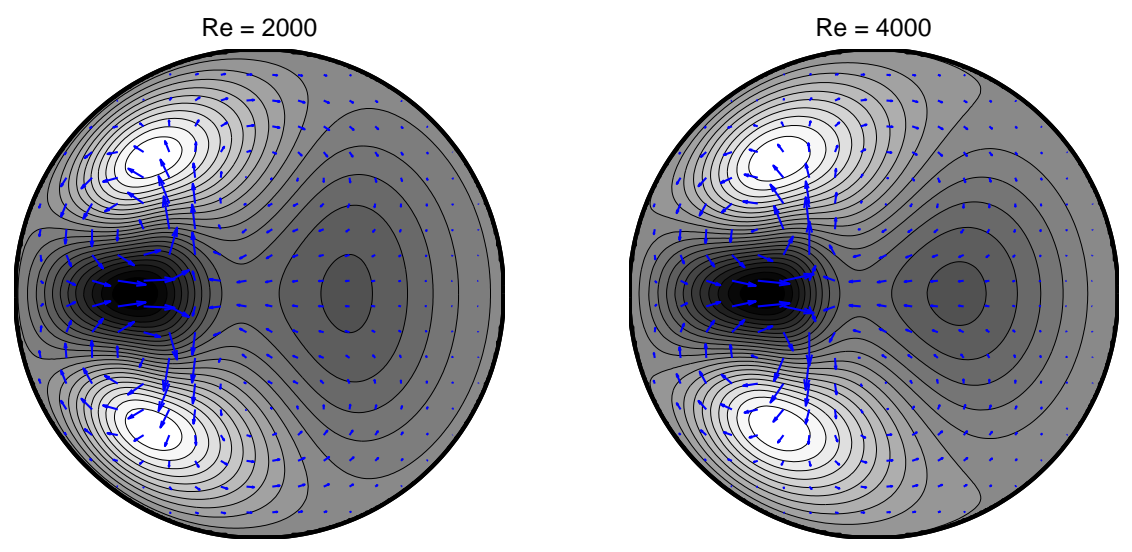

FiguRE 1. Contour plots of $z$-averaged streamwise velocity with the laminar flow subtracted. Rolls are superposed. The contour levels are equispaced in the intervals $[-0.195,0.170]$ and $[-0.191,0.148]$, respectively, with the lighter regions being faster. The maximum magnitude of the vectors in superposed quiver plots are 0.0075 and 0.0038 , respectively.

Another point to be mentioned is that puffs, in which streak patterns resembling those of some traveling waves have been detected, are observed experimentally only for $R e<2800$. The transition experiments that measure thresholds (Hof et al. 2003; Peixinho and Mullin 2007) reach Re as high as 20000. Thus the relevance of puffs to transition may seem limited. However, there is a possibility that puffs exist as solutions of the Navier-Stokes equation beyond the Re at which they are observed in experiments (Willis and Kerswell 2009).

Most of the lower branch solutions of pipe flow and the channel flows seem to be on the laminar-turbulent boundary (Duguet et al. 2008; Gibson et al. 2008; Itano and Toh 2001; Kawahara 2005; Kerswell 2005; Kerswell and Tutty 2007; Schneider et al. 2008; Viswanath 2008a; Wang et al. 2007), which means that for some tiny disturbances of the lower branch solution, the disturbed state evolves and becomes laminar uneventfully. For other disturbances, the disturbed stated evolves and becomes turbulent, or undergoes a turbulent episode before it becomes laminar.

In this article, we investigate if there are small disturbances of the laminar solution, for which the disturbed state evolves and hits a given lower branch solution. By small, we mean firstly that the magnitude of the disturbance should decrease algebraically with $R e$ and secondly that the disturbance should not change the mean flow significantly. The existence of such a disturbance would establish that the flow can transition from laminar to turbulence by passing through the vicinity of the given lower branch traveling wave.

In fact, Kreiss et al. (1994) found such disturbances when computing thresholds without fully realizing that they were hitting a lower branch equilibrium solution of plane Couette flow. But the situation they tackled is an especially simple one because the lower branch solution has a single unstable direction (Schneider et al. 2008; Toh and Itano 2003; Viswanath 2008a; Wang et al. 2007). We consider the asymmetric traveling wave computed by Pringle and Kerswell (2007). That traveling wave has two unstable directions both of which lie in a symmetric subspace, and thus serves to illustrate that the method used for computing thresholds cannot be used to hit traveling waves that have more than one unstable direction.

The asymmetric traveling wave of Pringle and Kerswell (2007), which has two fast streaks located near one side of the pipe, is shown in Figure 1. The preferential location of the streaks towards one side is also found in edge states that occur in transition 


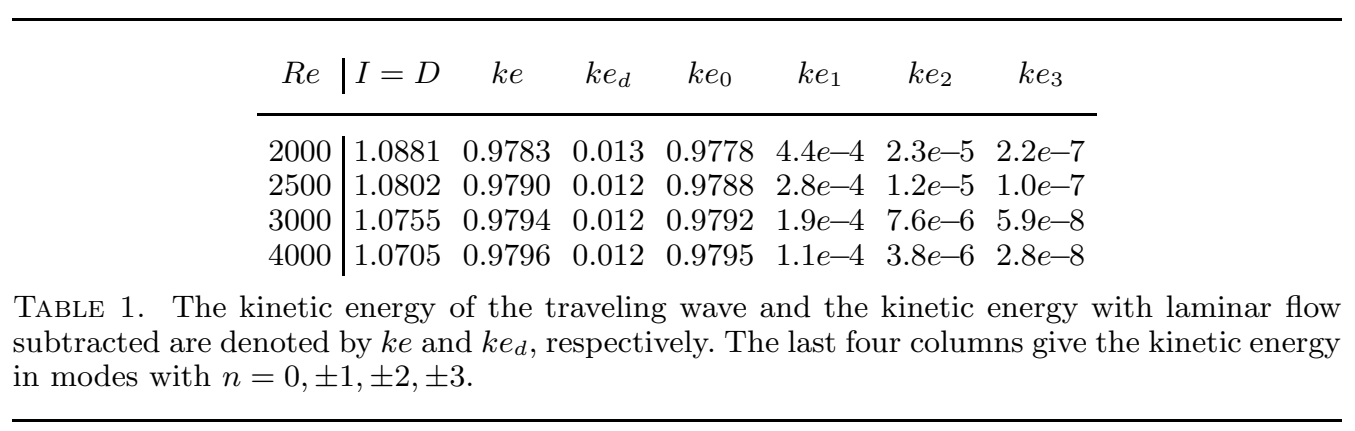

computations (Schneider et al. 2007b). Table 1 gives basic data for that traveling wave at four different Re. That data will be useful for judging the closeness of approaches to the traveling wave. The choice of units, the significance of $I$ and $D$ in Table 1 , and the meaning of the streamwise modes with $n=0, \pm 1, \pm 2, \pm 3$ are explained in Section 2. More extensive data for the asymmetric traveling wave can be found elsewhere (Viswanath 2008b).

To find a small disturbance of the laminar solution that evolves into a given lowerbranch state, it is necessary to consider a linear superposition of disturbances whose dimension equals that of the unstable manifold of the lower-branch state. That requirement follows from a consideration of the co-dimension of the stable manifold of the lower-branch state. In addition, the disturbances that are linearly combined must be chosen carefully.

To hit the asymmetric traveling wave, we consider three different disturbances of the laminar solution. The first disturbance is obtained by extracting the rolls, which are formed by averaging the traveling wave in the streamwise direction and retaining only the radial and azimuthal components of the velocity field. The choice of rolls is related to the so-called lift-up mechanism (Landahl 1980). The two other disturbances are the two unstable eigenvectors of the traveling wave. If one of the eigenvectors is added to the traveling wave, it has the effect of either reinforcing or weakening the fast streaks. The other eigenvector seems to alter the location of the fast streaks. It must be remembered, however, that the disturbances are added to the laminar solution and not to the traveling wave.

In Section 4, we show that disturbances of the laminar flow obtained by varying any two of these three disturbances evolve and hit the traveling wave. The choice of the unstable eigenvectors might seem puzzling as our intention is to hit the traveling wave and not to move away from it. The reason that choice works is partially explained in Sections 3 and 4 . Section 4 also shows that the magnitudes of the disturbances needed to hit the asymmetric traveling wave diminish algebraically with $R e$.

All our computations use a pipe that is $\pi$ pipe diameters long and the traveling waves are computed with 85715 active degrees of freedom. The computations of relative periodic solutions (or modulated traveling waves) in plane Couette flow use triple the number of degrees of freedom (Viswanath 2007), although those computations are roughly 5 to 10 times as expensive with the same number of degrees of freedom. The computation of traveling waves is an insignificant part of the total computational expense, however, as will become clear in Section 4. We need to use a short pipe to keep the total computational expense manageable.

The pipe we use is too short to capture transitional structures such as puffs. To add to the earlier discussion of the relevance of short pipe computations of traveling waves, 
we mention the work of Mellibovsky and Meseguer (2006) which seems to suggest that transition scenarios can be independent of pipe length. The logic which is used to find disturbances of the laminar solution that hit the asymmetric traveling wave makes fairly intricate use of the dynamical properties of the traveling wave. More work is needed to determine if the same logic is applicable to transition in pipes of more realistic length.

\section{Preliminaries}

The code for direct numerical simulation of pipe flow uses cylindrical coordinates with $u, v$, and $w$ being the components of the velocity in the radial $(r)$, polar $(\theta)$, and axial $(z)$ directions, respectively. The boundary conditions are no-slip at the walls and periodic in the $z$ direction with constant mass-flux. The length of the periodic domain in the $z$ direction is denoted by $2 \pi \Lambda$. We use $\Lambda=1$ throughout. The units for distance and velocity are chosen so that the pipe radius is 1 and the Hagen-Poiseuille profile is given by $w=1-r^{2}$. The Reynolds number $R e$ is based on the pipe radius, centerline velocity of the Hagen-Poiseuille flow, and kinematic viscosity $\nu$. The unit of mass is chosen so that the density of the fluid is 1 . The units and boundary conditions follow those of Faisst and Eckhardt (2004).

Let $\bar{w}(r)$ denote the mean velocity in the axial direction, and $\bar{v}(r)$ the mean velocity in the polar direction. The mass-flux per unit area is given by $2 \int_{0}^{1} r \bar{w}(r) d r$ and is equal to $1 / 2$ for all velocity fields that obey the boundary condition. The pressure gradient necessary to maintain constant mass-flux varies from instant to instant. For the HagenPoiseuille flow, it is $-4 / R e$.

The spatial discretization is spectral. The radial component of the velocity $u$ is represented as

$$
u(r, \theta, z)=\sum_{n=-N}^{n=N} \sum_{m=-M}^{m=M} \hat{u}_{n, m}(r) \exp (i m \theta) \exp (i n z / \Lambda) .
$$

For the velocity field to be regular at $r=0$, the coefficients $\hat{u}_{n, m}(r)$ must be even functions of $r$ for odd $m$ and odd functions of $r$ for even $m$. Thus the functions can be reconstructed by storing their values at $r=\cos (i \pi / L), i=0,1, \ldots,(L-1) / 2$. We assume $L$ odd so that there is no point at $r=0$ (Trefethen 2000). The radial component of vorticity is denoted by $\xi$. It is represented in the same way $u$ is represented. The other quantities used to represent the velocity field are $\bar{v}(r)$, which is an odd function of $r$, and $\bar{w}(r)$ which is an even function of $r$. The velocity field is constructed using $u, \xi, \bar{v}, \bar{w}$ and the divergence free condition. The advection term was dealiased using the Orszag $3 / 2$ rule. All the computations use $(N, M, L)=(16,18,81)$.

The rate of energy dissipation per unit mass is given by $2 D / R e$, where $D$ is the integral of

$$
\frac{1}{4 \pi^{2} \Lambda}\left(\frac{1}{r^{2}}\left(u^{2}+v^{2}-2 \frac{\partial u}{\partial \theta} v+2 u \frac{\partial v}{\partial \theta}\right)+\sum_{U=u, v, w}\left(\frac{\partial U}{\partial r}\right)^{2}+\left(\frac{\partial U}{\partial z}\right)^{2}+\frac{1}{r^{2}}\left(\frac{\partial U}{\partial \theta}\right)^{2}\right)
$$

over the volume of the pipe. In its more familiar form, $D$ is the integral of the sum of the norms of gradients of the three components of the velocity field (Wedin and Kerswell 2004). The term under the summation in (2.2) gives $|\nabla U|^{2}$ for a scalar field $U(r, \theta, z)$. The terms outside the summation in (2.2) arise as cross terms when that operator is applied to $u \cos \theta-v \sin \theta$ and $u \sin \theta+v \cos \theta$. The explicit form of (2.2) displays the $1 / r^{2}$ singularities that are hidden in vector notation. Because those singularities cancel at $r=0$, the numerical evaluation of $D$ in a spectral code is a delicate matter. The rate 
of energy input per unit mass is given by $2 I / R e$, where

$$
I=-\frac{R e}{4 \pi^{2} \Lambda} \int \nabla \cdot(p \mathbf{u}),
$$

with $p$ being pressure and with the integral being over the volume of the pipe. For the Hagen-Poiseuille laminar flow, both $D$ and $I$ evaluate to 1 .

Figure 1 shows the asymmetric traveling wave solution first computed by Pringle and Kerswell (2007). To compute that traveling wave, we added rolls which approximate the pattern in Figure 1 to the laminar solution and evolved the velocity field to allow the streaks to develop. The resulting velocity field was used as the initial guess for the GMRES-hookstep method, which converged without a hitch. The number of active degrees of freedom in the representation of a velocity field is $(L-2)+((2 N-1)(2 M-1)-1)(L-3) / 2$. The method uses translation operators to handle the invariance of the pipe-flow equation with respect to shifts along $z$ and rotations along $\theta$. These operators are given by

$$
\begin{aligned}
& \mathcal{T}_{1} u(r, \theta, z)=\sum_{m, n} i m \hat{u}_{n, m}(r) \exp (i m \theta) \exp (i n z / \Lambda) \\
& \mathcal{T}_{2} u(r, \theta, z)=\sum_{m, n}(i n / \Lambda) \hat{u}_{n, m}(r) \exp (i m \theta) \exp (i n z / \Lambda),
\end{aligned}
$$

where the indices $m, n$ correspond to the representation (2.1). A detailed description of the GMRES-hookstep method can be found elsewhere (Viswanath 2007, 2008b).

The equations of pipe flow are unchanged by the shift-reflect symmetry:

$$
\begin{gathered}
u(r, \theta, z) \rightarrow u(r,-\theta, z+\pi \Lambda) \\
v(r, \theta, z) \rightarrow-v(r,-\theta, z+\pi \Lambda) \\
w(r, \theta, z) \rightarrow w(r,-\theta, z+\pi \Lambda) .
\end{gathered}
$$

The velocity field of the traveling wave of Figure 1 is also unchanged by this discrete symmetry.

The magnitudes of disturbances and the norms of velocity fields are given in Section 4 and other places using the square root of kinetic energy norm. The kinetic energy, which is reported in tables such as Table 1, is normalized to be 1 for laminar flow.

To conclude this section, we mention a technical point about pipe flow simulation using spectral codes that appears not to have been discussed in the literature. Once the advection term is computed, the equations for evolving the modes decouple for pairs $(m, n)$ such that the resulting equations depend only upon $r$ for a fixed $(m, n)$. The decoupled equations will have terms with the factor $m^{2} / r^{2}+n^{2} / \Lambda^{2}$ in the denominator, and because of that factor the terms will have a singularity at the point $r=-i m \Lambda / n$ in the complex plane. If the number $2 N$ of grid points in the streamwise direction is increased while keeping the pipe length $2 \pi \Lambda$ fixed, that singularity moves closer to the real line with greater values of $n$ now being allowed. When the singularity moves closer to the real line, one has to use more grid points in the $r$ direction to solve the decoupled equations with the same level of accuracy (Trefethen 2000).

\section{Unstable manifold of the traveling wave}

To find disturbances of the laminar solution that evolve and hit the asymmetric traveling wave, it is essential to understand the unstable directions and the unstable manifold of that traveling wave. Suppose we disturb the laminar solution using rolls of the ap-

propriate form and some "noise", the magnitude of which is a fixed fraction of that of 


\begin{tabular}{c|ccc}
$R e$ & $\lambda_{1}$ & $\lambda_{2}$ & $\lambda_{3}$ \\
\hline 2000 & 0.03247 & 0.00897 & -0.00594 \\
2500 & 0.03049 & 0.00725 & $-0.02282+i 0.02041$ \\
3000 & 0.02861 & 0.00631 & $-0.01978+i 0.01664$ \\
4000 & 0.02529 & 0.00531 & $-0.01536+i 0.01190$
\end{tabular}

TABLE 2. $\quad \lambda_{1}$ and $\lambda_{2}$ are the only unstable eigenvalues. $\lambda_{3}$ has the greatest real part among the stable eigenvalues whose eigenvectors lie in the shift-reflect invariant subspace.
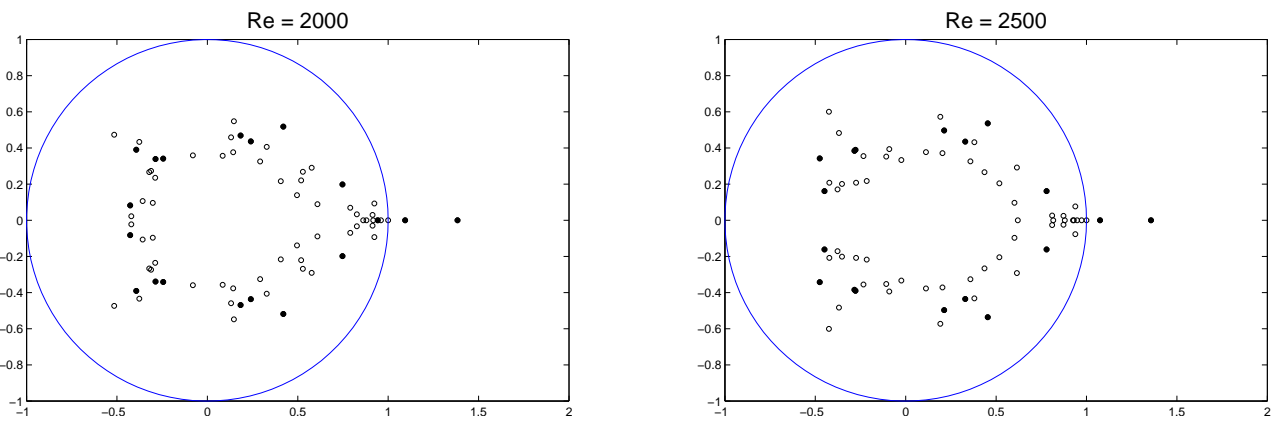

Figure 2. For eigenvalues $\lambda$ of the traveling wave, the plots show $\exp (10 \lambda)$ as circles for easier visualization. If the corresponding eigenvector lies in the shift-reflect invariant subspace, the circle is solid.

the rolls, to introduce streamwise dependence. The disturbed state will evolve and develop streaks. At the point of closest approach to the traveling wave, we can think of the evolving velocity field as the traveling wave plus two components, one of which is a combination of the stable eigenvectors of the traveling wave with the other being a combination of the unstable eigenvectors. The stable eigenvectors will decay under evolution. However, the component along the unstable eigenvectors will be amplified and will take the evolving velocity field away from the traveling wave. To ensure that the disturbed state hits the traveling wave, the disturbance has to be arranged in such a way that the evolving velocity field is free of the unstable directions as it approaches the traveling wave.

Such a disturbance is easiest to arrange, if the traveling wave has only one unstable direction. The component along that direction at the point of closest approach can be eliminated by simply varying the magnitude of the initial disturbance. However, the asymmetric traveling wave has two unstable directions as shown in Table 2. The two unstable eigenvalues $\lambda_{1}$ and $\lambda_{2}$ decrease with increasing $R e$ at rates given by $R e^{-0.41}$ and $R e^{-0.87}$, respectively (Viswanath 2008b). Table 2 also shows the leading stable eigenvalue.

Figure 2 gives a more complete idea of the spectrum of the linearization around the asymmetric traveling wave. The spectra at different $R e$ were computed using the Arnoldi iteration. Some of the interior eigenvalues near the centers of the circles in Figure 2 are omitted. But we are certain that no unstable eigenvalues are omitted. In addition, we have verified that none of the eigenvalues in the figure is spurious.

Data for the unstable eigenvectors is given in Table 3. Most of the kinetic energy of the traveling waves themselves is in the $n=0$ (or mean) mode, as shown in Table 1. Much of the kinetic energy remains in $n=0$ for the $\lambda_{1}$ eigenvector, although $n=1$ now 


\begin{tabular}{c|cccc|cccc}
$R e$ & $k e_{0}$ & $k e_{1}$ & $k e_{2}$ & $k e_{3}$ & $k e_{0}$ & $k e_{1}$ & $k e_{2}$ & $k e_{2}$ \\
\hline 2000 & $6.9 e-1$ & $2.6 e-1$ & $4.9 e-2$ & $1.1 e-3$ & $1.2 e-1$ & $7.4 e-1$ & $1.4 e-1$ & $3.1 e-3$ \\
2500 & $7.0 e-1$ & $2.6 e-1$ & $4.2 e-2$ & $7.6 e-4$ & $1.1 e-1$ & $7.7 e-1$ & $1.2 e-1$ & $2.3 e-3$ \\
3000 & $7.0 e-1$ & $2.6 e-1$ & $3.8 e-2$ & $6.2 e-4$ & $1.1 e-1$ & $7.8 e-1$ & $1.1 e-1$ & $1.9 e-3$ \\
4000 & $7.0 e-1$ & $2.6 e-1$ & $3.2 e-2$ & $4.9 e-4$ & $1.1 e-1$ & $7.9 e-1$ & $9.4 e-2$ & $1.5 e-3$
\end{tabular}

TABLE 3. The kinetic energies in the $n=0, \pm 1, \pm 2, \pm 3$ modes of the $\lambda_{1}$ and $\lambda_{2}$ eigenvectors. The eigenvectors are normalized to have kinetic energy equal to 1 .
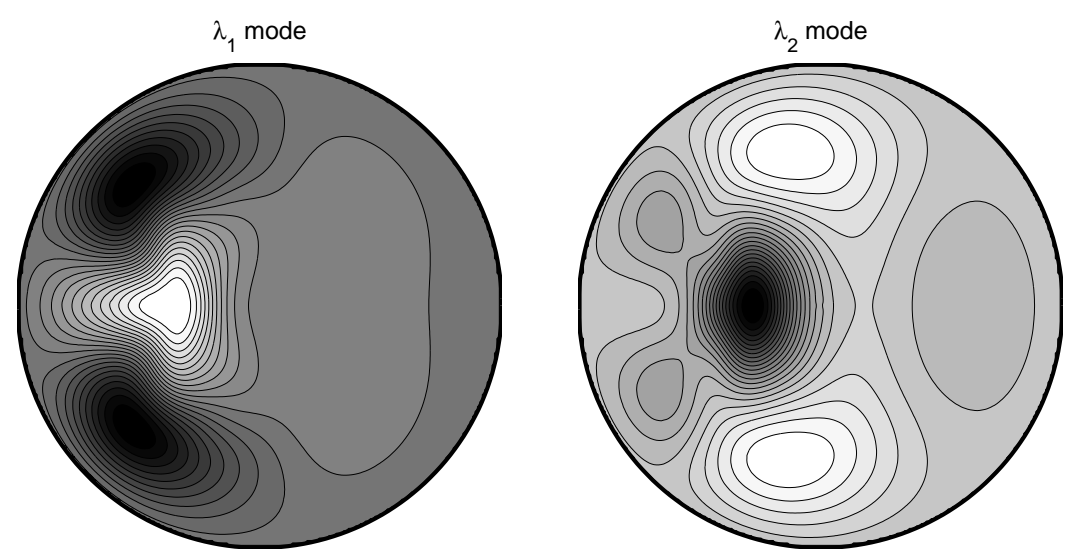

FIGURE 3. Contour plots of the $z$-averaged streamwise velocity for the two unstable eigenvectors at $R e=2500$. If the eigenvectors are normalized to have unit kinetic energy, the level curves are equispaced in the intervals $[-1.38,1.66]$ and $[-1.01,0.34]$, respectively. The lighter regions correspond to higher values. At other values of $R e$, the signs of the eigenvectors are chosen to yield plots similar to the ones above.

has more than a quarter of the kinetic energy. For the $\lambda_{2}$ eigenvector, the $n=1$ mode dominates.

Figure 3 shows that the $\lambda_{1}$ eigenvector weakens the high speed streaks of the traveling wave. The effect of adding the $\lambda_{2}$ eigenvector to the traveling wave would be to displace the high speed streaks to a more symmetrical position. It must be noted, however, that the plot of the streaks of the $\lambda_{2}$ eigenvector is not as meaningful because the $n=1$ mode is dominant. The two plots in Figure 3 are used to assign positive and negative signs to the eigenvectors at different $R e$ in a consistent manner. When comparing cross-sections of velocity fields to traveling wave solutions (Eckhardt and Schneider 2008; Hof et al. 2004; Willis and Kerswell 2008), it may be worthwhile to look at the unstable eigenvectors of the traveling waves. The inevitable deviations from the streak patterns of the traveling waves may correlate with the streak patterns of the unstable eigenvectors.

Figure 4 shows the mean streamwise flow that corresponds to the $\lambda_{1}$ and $\lambda_{2}$ eigenvectors. To form an idea of the distortion to the mean flow of the laminar solution when those eigenvectors are added as disturbances, the plots in Figure 4 must be scaled by a factor of $1 / 50$ or less.

Figure 5a shows that if the asymmetric traveling wave is disturbed with a small and positive multiple of the $\lambda_{1}$ eigenvector, the disturbed state evolves and becomes laminar uneventfully. That is unsurprising because the disturbance has the effect of weakening 

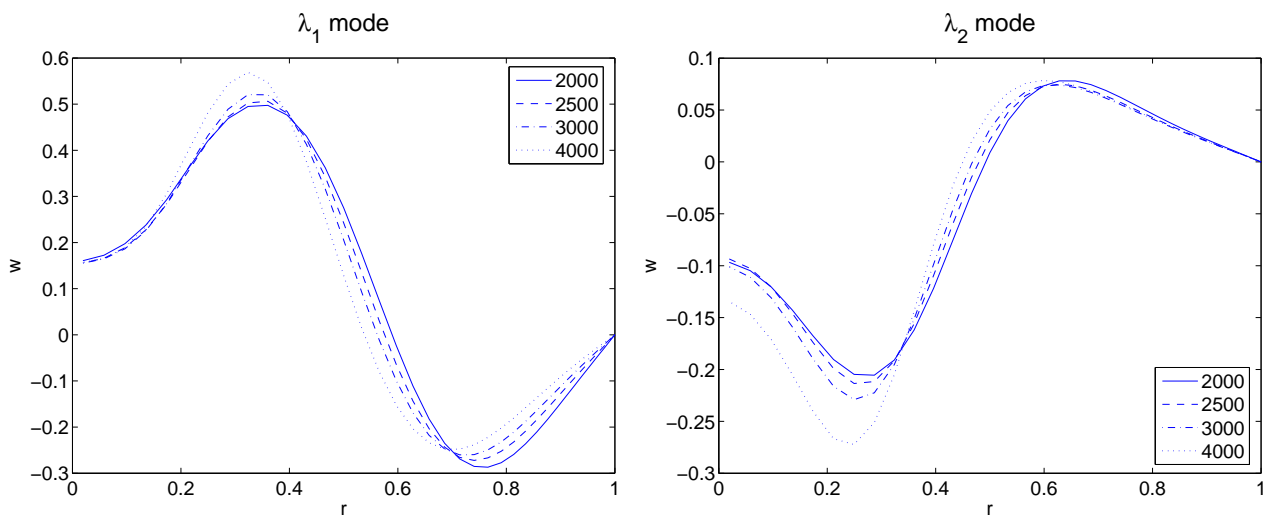

FiguRE 4. Plots of the mean streamwise flow $\bar{w}(r)$ at various values of $R e$. The eigenvectors are normalized to have kinetic energy equal to 1.

(a)
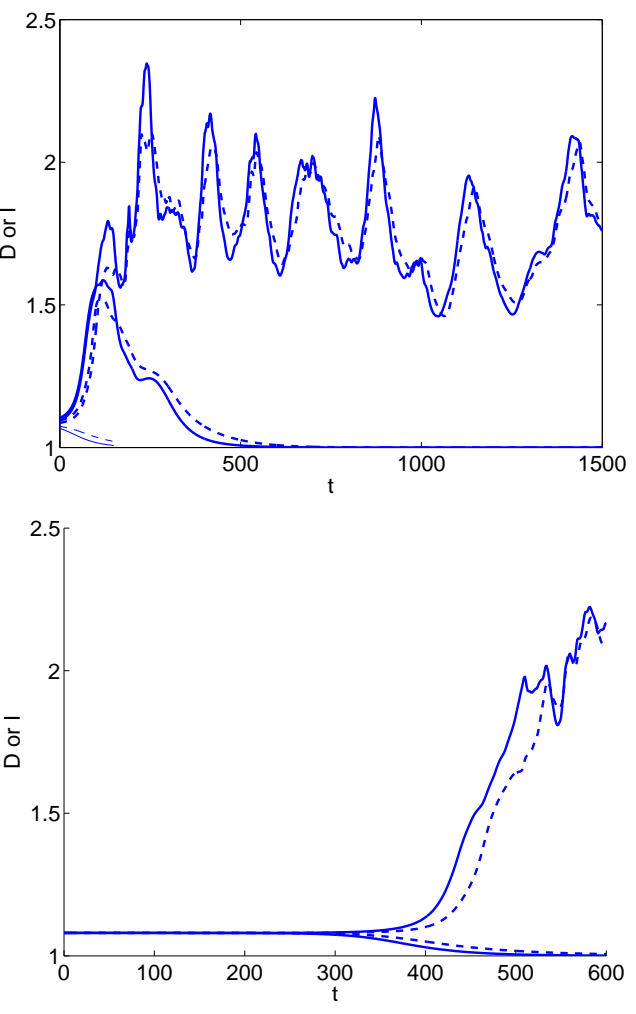

Figure 5. (a) Plots of D (solid) and I (dashed) against time at $R e=2000$ and $R e=2500$. For both values of $R e$, perturbation of the traveling wave by a positive multiple of the $\lambda_{1}$ eigenvector shown in Figure 4 leads to rapid laminarization (thin lines at lower left corner). Perturbation by a negative multiple leads to a long transient at $R e=2000$ and what appears to be sustained turbulence at $R e=2500$. (b) Plots close to the edge.

the high speed streaks. In contrast, adding a negative multiple leads to what appears to be sustained turbulence at $R e=2500$. It is easily noticeable that energy dissipation $D$ is greater than energy input $I$ when the plots in Figure 5a spike up, but is lesser when the plots dip down. Thus the kinetic energy of the velocity field as a whole decreases during 


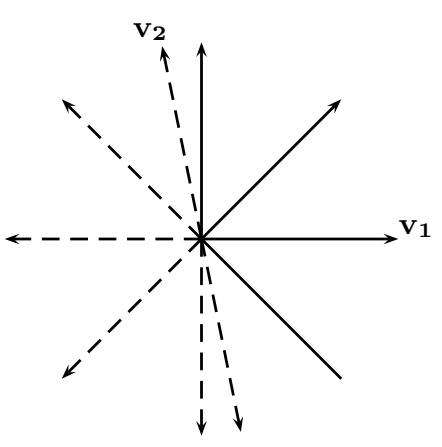

(a)

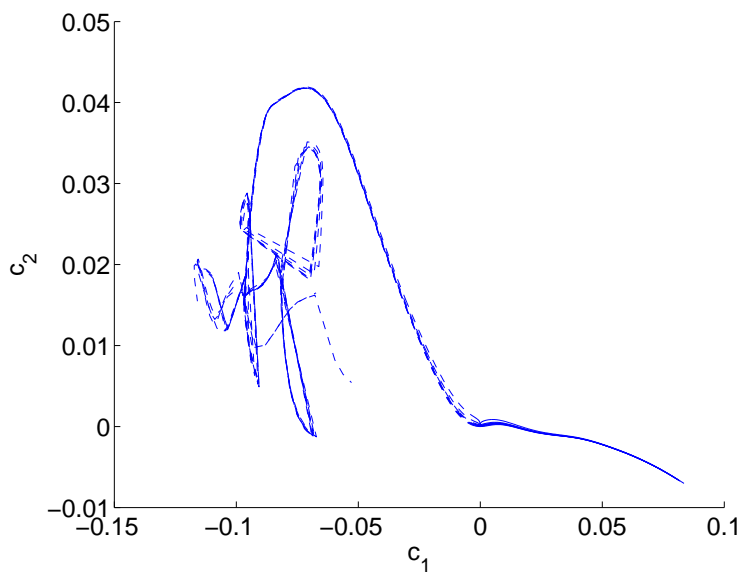

FiguRE 6. (a) A schematic sketch of directions on the unstable manifold, where the two eigenvectors are labeled. The trajectories initiated in the dashed directions undergo turbulent episodes. (b) Projections of trajectories whose initial points are obtained by perturbing the traveling wave at $R e=2500$ by a combination of the $\lambda_{1}$ and $\lambda_{2}$ modes. The initial points are all near $(0,0)$. The trajectories that laminarize turn right, while those that transition to turbulence (dashed) turn left.

the spikes, but increases during the dips. The decrease of kinetic energy during a spike is well correlated with flattening of the mean velocity profile.

Figure 6a shows a schematic sketch of the unstable directions of the asymmetric traveling wave, while distinguishing between directions that turn turbulent and ones that do not. Figure $5 \mathrm{~b}$ corresponds to two trajectories close to the border between turbulent and laminar directions. In that figure, the trajectories near the border separate after $t=300$. By refining the border, it appears that the point of separation can be deferred indefinitely with a view to locating edge states.

To better visualize the unstable manifold, we adopt a technique introduced by Gibson et al. (2008) with the aim of getting good phase space visualizations of turbulent trajectories. The velocity field is a point in phase space and the evolution of an initial velocity field with respect to the incompressible Navier-Stokes equation is a trajectory in that phase space. Because the phase space is infinite-dimensional and does not lend itself to plots directly, one has to use projections. An obvious projection would be to pick some FourierChebyshev modes from the discretization of the velocity field. Although such projections have been employed, they have a number of shortcomings. The choices of the component of the velocity vector and of the mode of that component are both arbitrary. The component and the mode that are chosen capture only a partial aspect of the velocity field. As a result of these shortcomings, such projections look messy and one cannot form a good idea of the dynamical structures in phase space from such projections.

Following (Gibson et al. 2008), the projection we use picks a set of velocity fields that appears well suited to visualize trajectories on the unstable manifold. Let $\mathbf{u}_{T W}$ be the velocity field of the traveling wave and let $\mathbf{u}^{\prime}$ and $\mathbf{u}^{\prime \prime}$ be an orthonormal basis for its unstable space. The notion of orthogonality between velocity fields corresponds to the kinetic energy norm. We will choose $\mathbf{u}^{\prime}$ to be the same direction as the leading eigenvector. With that choice the second eigenvector at $R e=2500$ is approximately $-0.13 \mathbf{u}^{\prime}+0.99 \mathbf{u}^{\prime \prime}$. For each velocity field that satisfies the shift-reflect symmetry (2.5), we obtain a projection in terms of $\mathbf{u}_{T W}, \mathbf{u}^{\prime}$, and $\mathbf{u}^{\prime \prime}$. The velocity fields $\mathbf{u}^{\prime}$ and $\mathbf{u}^{\prime \prime}$ satisfy the no-slip boundary condition and have zero mass-flux. 


\begin{tabular}{|c|c|c|c|c|c|c|c|c|}
\hline$f_{r}$ & $f_{1}$ & $f_{2}$ & $T$ & $\delta$ & $k e_{0}$ & $k e_{1}$ & $k e_{2}$ & $k e_{3}$ \\
\hline $7.084220 e-3$ & $-2.114944 e-2$ & 0 & 371.49 & $8.1 e-4$ & $9.8 e-1$ & $4.3 e-4$ & $2.2 e-5$ & $2.1 e-7$ \\
\hline $1.242439 e-2$ & $1.890000 e-2$ & 0 & 219.70 & $8.9 e-3$ & $9.8 e-1$ & $3.3 e-4$ & $1.4 e-5$ & $1.0 e-7$ \\
\hline $9.119378 e-3$ & 0 & $1.720663 e-2$ & 257.80 & $4.2 e-3$ & $9.8 e-1$ & $4.1 e-4$ & $2.0 e-5$ & $1.9 e-7$ \\
\hline $1.017473 e-2$ & 0 & $-1.400000 e-2$ & 240.90 & $5.5 e-3$ & $9.8 e-1$ & $3.7 e-4$ & $1.7 e-5$ & $1.4 e-7$ \\
\hline $9.048182 e-3$ & $3.923814 e-4$ & $1.757170 e-2$ & 281.40 & $2.9 e-3$ & $9.8 e-1$ & $4.2 e-4$ & $2.2 e-5$ & $2.0 e-7$ \\
\hline $5.687034 e-3$ & $-1.643322 e-2$ & 0 & 323.84 & $2.9 e-3$ & $9.8 e-1$ & $2.5 e-4$ & $1.0 e-5$ & $7.7 e-8$ \\
\hline $9.591329 e-3$ & $1.562075 e-2$ & 0 & 318.60 & $7.7 e-3$ & $9.8 e-1$ & $3.1 e-4$ & $1.5 e-5$ & $1.4 e-7$ \\
\hline $7.049209 e-3$ & 0 & $1.420195 e-2$ & 268.00 & $4.8 e-3$ & $9.8 e-1$ & $2.4 e-4$ & $9.4 e-6$ & $6.9 e-8$ \\
\hline $7.986395 e-3$ & 0 & $-1.115761 e-2$ & 309.80 & $6.0 e-3$ & $9.8 e-1$ & $2.2 e-4$ & $8.1 e-6$ & $5.5 e-8$ \\
\hline $5.676511 e-3$ & $-1.646355 e-2$ & $8.768074 e-5$ & 292.70 & $3.7 e-3$ & $9.8 e-1$ & $2.9 e-4$ & $1.3 e-5$ & $1.2 e-7$ \\
\hline
\end{tabular}

TABle 4. Data at $R e=2000$ (above the double line) and at $R e=2500$ (below the double line). The $f_{\mathrm{s}}$ are magnitudes of disturbances of the laminar solution. $T$ is the time of closest approach to the traveling wave. $\delta$ is the distance from the traveling wave at that time. The last four columns give the kinetic energies in the $n=0, \pm 1, \pm 2, \pm 3$ modes at the point of closest approach.

Given a velocity field $\mathbf{u}$ that satisfies the shift-reflect symmetry, one can decompose it as $\mathbf{u}-\mathbf{u}_{T W}=c_{0} \mathbf{u}^{\prime}+c_{1} \mathbf{u}^{\prime \prime}+\mathbf{r}$, where the remainder $\mathbf{r}$ is orthogonal to the plane of the eigenvectors. One could use $c_{0}$ and $c_{1}$ to represent $\mathbf{u}$ in a plot, but that would be unsatisfactory. The problem is that one can translate $\mathbf{u}_{T W}$ in the streamwise direction and obtain different velocity fields that stand for the same wave. In order to eliminate dependence on translations in the $z$ direction, we shift the velocity field $\mathbf{u}$ by $s_{z}$ in the $z$ direction and consider

$$
\mathbf{u}\left(r, \theta, z+s_{z}\right)-\mathbf{u}_{T W}=c_{0} \mathbf{u}^{\prime}+c_{1} \mathbf{u}^{\prime \prime}+\mathbf{r}_{s_{z}} .
$$

The shift $s_{z}$ is chosen to minimize $\left\|\mathbf{r}_{s_{z}}\right\|$, and the axes of the projection, $c_{0}$ and $c_{1}$, are the coefficients for that shift. The need to pick a shift $s_{z}$ arises because the equations of pipe-flow are unchanged by translations along $z$. The shift-reflect symmetry is broken by rotations in the $\theta$ direction. Since we have restricted ourselves to vector fields with the shift-reflect symmetry, shifts in $\theta$ are not considered in (3.1). The need to factor out continuous symmetries arises in ODEs (Gilmore and Letellier 2007) and PDEs such as the Kuramoto-Sivashinky equation as well (Cvitanović et al. 2007).

Figure $6 \mathrm{~b}$ shows trajectories on the unstable manifold using such a projection. The initial velocity fields were of the form $\mathbf{u}_{T W}+\epsilon a \mathbf{u}^{\prime}+\epsilon b \mathbf{u}^{\prime \prime}$, with $\epsilon=\times 10^{-4}$ and $a, b$ being scalars. In all, we considered ten velocity fields corresponding to $(a, b)=( \pm 1, \pm 1)$, the four coordinate directions, and two directions along the second eigenvector. Since $\epsilon$ is small, all these velocity fields were very nearly on the unstable manifold. The distinction between trajectories that laminarize uneventfully and those that undergo a turbulent episode is clear in Figure 6b.

In the next section, we return to such projections of the unstable manifold to partially justify arguments used to find disturbances of the laminar flow which evolve and hit the asymmetric traveling wave.

\section{Hitting the traveling wave at $R e=2000$ and $R e=2500$}

The $f_{r}$ column of Table 4 gives the norm of the rolls. The velocity field of the rolls, denoted by $\mathbf{u}_{r}$, is obtained by averaging the traveling wave in the streamwise direction and discarding the streamwise component of the velocity. The norms of the unstable 
eigenvectors are denoted by $f_{1}$ and $f_{2}$. The velocity fields of the eigenvectors are denoted by $\mathbf{u}_{1}$ and $\mathbf{u}_{2}$. The laminar solution is $\mathbf{u}_{L}$ and the traveling wave is $\mathbf{u}_{T W}$.

The distance $\delta$ of closest approach listed in Table 4 is obtained as follows. The NavierStokes equation is integrated from the initial velocity field $\mathbf{u}_{L}+f_{r} \mathbf{u}_{r}+f_{1} \mathbf{u}_{1}+f_{2} \mathbf{u}_{2}$. The initial velocity field has the shift-reflect symmetry and so does $\mathbf{u}_{t}$, where $\mathbf{u}_{t}$ is the velocity field at time $t$. Define

$$
\delta\left(f_{r}, f_{1}, f_{2}\right)=\min _{t \geq 0} \min _{0 \leq s_{z}<2 \pi \Lambda}\left\|\mathbf{u}_{t}\left(r, \theta, z+s_{z}\right)-\mathbf{u}_{T W}\right\| .
$$

To compare $\mathbf{u}_{t}$ and $\mathbf{u}_{T W}$, one has to minimize over shifts $s_{z}$ for the same reason as in (3.1). To find the minimizing shift $s_{z}$, we first try $s_{z}=\pi \Lambda k / N, 0 \leq k<2 N$. Using that data, an interval that contains the minimum is found and that interval is refined recursively to a depth equal to 30 . We refer to the result of the inner minimum in (4.1) as the distance between $\mathbf{u}_{T W}$ and $\mathbf{u}_{t}$. This method of finding that distance is expensive, with the cost of finding the distance being more than 20 times the cost of a single time step. However, it finds the distance with an accuracy of 4 or 5 digits.

Given the expense of finding the distance between $\mathbf{u}_{t}$ and $\mathbf{u}_{T W}$, the distance being the inner minimum in (4.1), care has to be exercised in finding the outer minimum over $t$. If the distance is computed after every time step, the cost of the computation becomes prohibitive. The wall time for integrating a velocity field for a time interval of 100 is about an hour on an Opteron processor, but becomes more than 20 hours if the distance to $\mathbf{u}_{\mathbf{T W}}$ is computed after every time step. For an initial waiting time when the streaks are still forming, we do not compute the distance at all. This waiting time is longer for larger Re. Thereafter the distance is computed every 100 time steps only, a time step being 0.01 . As the distances vary smoothly as a function of time, we use polynomial extrapolation to predict if the distance function has a minimum within the next 100 time steps or not. If it is predicted to have a minimum within the next 100 time steps, we measure the distance every 10 time steps. If the distance function is predicted to have a minimum within the next 10 time steps, we measure the distance after every time step. The value of $\delta$ is the first local minimum found in this manner and it is very probably also the global minimum over $t$. The time step can be successively decreased to get finer estimates of $\delta$, but that was not implemented.

The times $T$ at which the minima were attained are given in Table 4. $T$ is measured with a precision of .01 in only two lines of that table. The measurements in the other lines have a precision of 0.1 . If the last four columns of that table are compared with the last four columns of Table 1, the comparison confirms that the approach to the traveling wave is closer when $\delta$ is smaller. Figure 7 leaves no room for doubt that the first disturbances listed for $R e=2000$ and $R e=2500$ in Table 4 evolve and hit the corresponding traveling waves. The smallest distance $\delta$ from the traveling wave is realized after time $T$, which is listed in Table 4. Figure 7 shows that the plots of the distances from the traveling wave and the laminar solution both become flat around $t=T$. The disturbance moves away from the laminar solution rapidly at $t=0$. In contrast, for heteroclinic connections there are two flat regions in $t$, which correspond to time spent in the neighborhoods of the invariant solutions joined by the heteroclinic connection (Halcrow et al. 2009).

We are yet to explain the method used to find the numbers $f_{r}, f_{1}$, and $f_{2}$ in Table 4 . With each of those disturbances to the laminar solution, the disturbed state lands close to the stable manifold of the traveling wave and evolves to make a close approach of small $\delta$ to the traveling wave. Each line Table 4 was obtained by minimizing $\delta\left(f_{r}, f_{1}, f_{2}\right)$ in different ways. The manner of minimization will now be described.

Although $\delta$ has three arguments corresponding to three disturbances, each minimiza- 


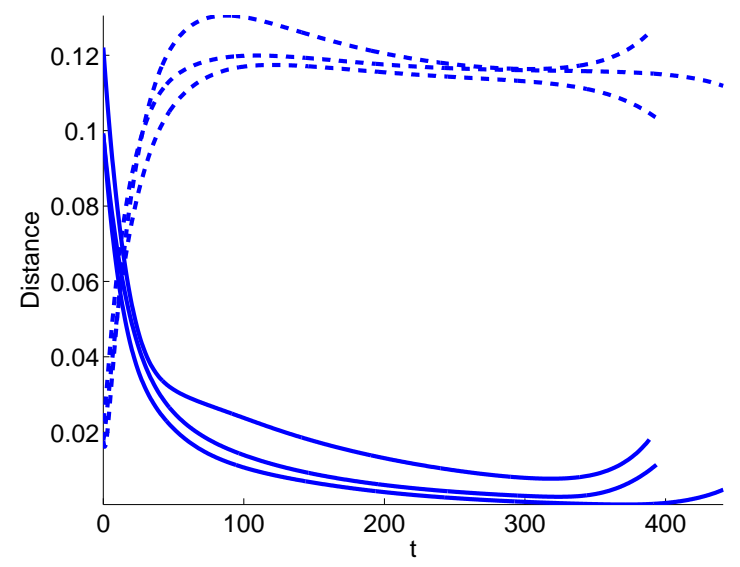

Figure 7. Distances from the laminar solution (dashed) and the traveling wave (solid). The three pairs of curves correspond to the first line with $R e=2000$ in Table 4 and the first two lines with $R e=2500$. Data in that table may be used to deduce the assignment of curves above to rows in that table.

tion was two dimensional. The unstable manifold of the traveling wave at the Re under consideration is two dimensional as shown by Table 2 . If the directions that correspond to translating and rotating the traveling wave are ignored, the co-dimension of the traveling wave is two. The inner minimization in (4.1) accounts for streamwise translations, while rotations around the pipe axis would break the shift-reflect symmetry. Thus the stable manifold is in effect a co-dimension two object.

Suppose there is some system of coordinates for the infinite-dimensional phase space in which the traveling wave is $(0,0,0, \ldots)$. Suppose further that its unstable manifold is given by fixing all except the first two coordinates at zero and its stable manifold is obtained by fixing the first two coordinates at zero. To disturb the laminar solution on to the stable manifold, the first two components must be zeroed out. Generically, it is impossible to zero out two components by varying the amplitude of a single disturbance. That is why we varied two disturbances.

All the rows of Table 4 have $f_{r}>0$. Adding the rolls to the laminar solution causes the flow to develop streaks of approximately the right form. But the key to hitting the traveling wave is to disturb the laminar state in such a way that the evolving velocity field is free from unstable directions as it approaches the traveling wave. If we were allowed to make disturbances near the traveling wave, we could simply eliminate the unstable directions. Since the disturbances are made to the laminar solution, we have to somehow guess the directions at $t=0$ which evolve into unstable directions at $t=T$, the time of closest approach. Analysis can possibly suggest a better choice, but we simply used the unstable directions $\mathbf{u}_{1}$ and $\mathbf{u}_{2}$ to disturb the laminar flow. In addition the magnitude of the rolls themselves can be varied to get a direction that has a nonzero component along the unstable manifold at $t=T$.

Each row of Table 4 that has $f_{1}=0$ or $f_{2}=0$ was gotten by fixing that disturbance at 0 and minimizing over the other two disturbances. In addition, the sign of the other disturbance that adds an eigenvector was prescribed. Thus there are four rows of that type for $R e=2000$ and $R e=2500$. For the last row with $R e=2000$ or $R e=2500, f_{r}$ was fixed while $f_{1}$ and $f_{2}$ were varied. Figure 8 shows that for disturbances at $R e=2500$, the flow evolves to a state where its dynamics is governed mainly by the unstable manifold of the traveling wave, thus partially supporting the reasoning used to find those disturbances. 


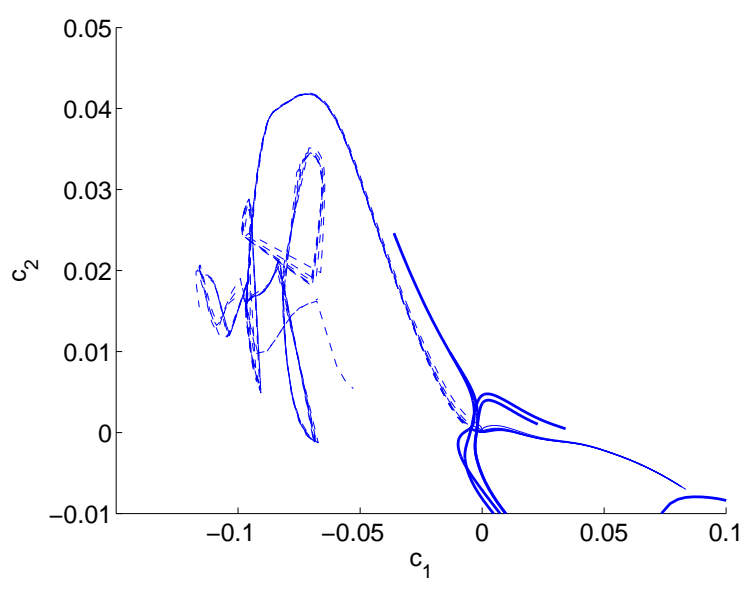

FiguRE 8. Similar to Figure $6 \mathrm{a}$, but the thick lines show the projections of trajectories at $R e=2500$ which are initialized using the disturbances in Table 4 .

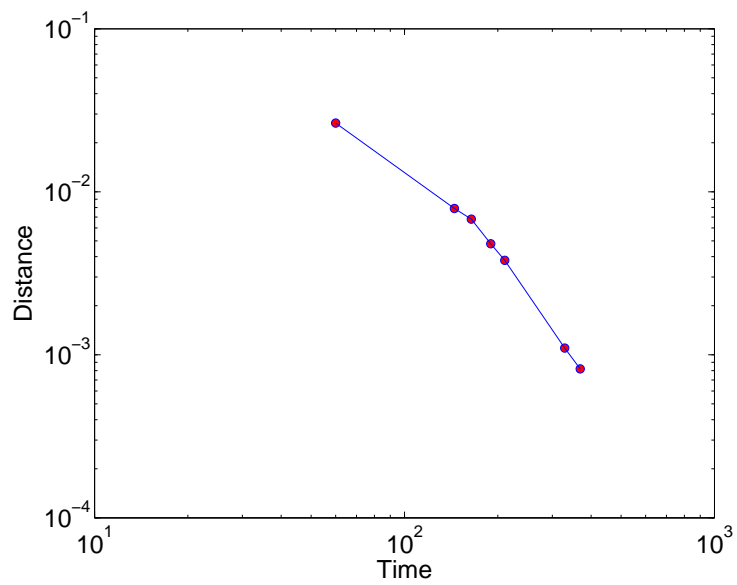

Figure 9. The axes correspond to the $T$ and $\delta$ columns in Table 4 . The plot is for $R e=2000$ and shows the progress of a single minimization (first line of Table 4). Each point corresponds to a certain stage in the sequence of optimizations used to find a disturbance of the laminar flow such that the disturbed flow evolves and hits the traveling wave.

When $\delta\left(f_{r}, f_{1}, f_{2}\right)$ is minimized numerically, the disturbances found at successive stages of the minimization give smaller $\delta$ but with larger values of $T$, the time of closest approach to the traveling wave, as shown in Figure 9. For the theoretical ideal $\delta=0, T$ would be infinite. Thus the numerical optimization becomes progressively more expensive.

A more severe impediment to numerical minimization is the non-smooth dependence of $\delta$ on the disturbances when $\delta=0$. Because the time to hit the traveling wave diverges, even a small change in the disturbances causes a big change in the value of $\delta$.

The numerical optimization was implemented using Matlab's $f$ mincon(), which allows constraints to be placed on the values of $f_{r}$ or $f_{1}$ or $f_{2}$. The $\mathrm{C}++$ code for computing the function $\delta\left(f_{r}, f_{1}, f_{2}\right)$ was invoked from Matlab. The unconstrained version $f$ minunc () was not used because it tends to take such large steps while varying $f_{r}$ or $f_{1}$ or $f_{2}$ that the numerical integration of the Navier-Stokes equation becomes unstable. Because of the non-smoothness, a nonlinear least squares solver, such as Matlab's lsqnonlin(), might be a better option than $f$ mincon(). lsqnonlin () minimizes $\sqrt{|x+2|}$ from $x=3$ with just 


\begin{tabular}{|c|c|c|c|c|c|c|c|c|c|}
\hline $\operatorname{Re} \mid$ & $f_{r}$ & $f_{1}$ & $f_{2}$ & $T$ & $\delta$ & $k e_{0}$ & $k e_{1}$ & $k e_{2}$ & $k e_{3}$ \\
\hline 2000 & $9.119378 e-3$ & 0 & $1.720663 e-2$ & 257.80 & $4.2 e-3$ & $9.8 e-1$ & $4.1 e-4$ & $2.0 e-5$ & $1.9 e-7$ \\
\hline 2500 & $7.049209 e-3$ & 0 & $1.420195 e-2$ & 268.00 & $4.8 e-3$ & $9.8 e-1$ & $2.4 e-4$ & $9.4 e-6$ & $6.9 e-8$ \\
\hline 3000 & $6.466047 e-3$ & 0 & $-9.890996 e-3$ & 356.61 & $7.0 e-3$ & $9.8 e-1$ & $2.2 e-4$ & $9.4 e-6$ & $8.4 e-8$ \\
\hline 4000 & $4.600000 e-3$ & 0 & $8.829559 e-3$ & 386.95 & $9.5 e-3$ & $9.8 e-1$ & $6.9 e-5$ & $1.5 e-6$ & $7.1 e-9$ \\
\hline
\end{tabular}

TABle 5. Data at various Re. The columns are as in Table 4.

6 function evaluations while fmincon() takes 63 function evaluations to find a slightly worse approximation to the minimum. It was not used, however, because it does not provide the facility to constrain the arguments.

The choice of initial guesses for the disturbances is not much of an issue because the numerical optimization is relatively efficient at the early stages. However, as $\delta=0$ is approached, the optimization routine tries unrealistically large steps, necessitating a lot of wasteful backtracking. It is difficult to assess the quality of the search directions. Each row in Table 4 required at least 200 hours of computing and often significantly more. The first rows with $R e=2000$ and $R e=2500$ required much more than 1000 hours to attain smaller values of $\delta$. The computations required repeated manual intervention to reset the parameters to $f \operatorname{mincon}()$, which is the reason we were able to run the numerical optimization longer for only two rows of Table 4.

Table 5 shows that the magnitude of the disturbances of the laminar flow required to hit the traveling wave diminishes with $R e$. The quality of the approach to the traveling wave degrades with increasing $R e$. The quality of the approach can be assessed using the $\delta$ column of Table 5 and by comparing the last four columns of that table to the last four columns of Table 1. We are not certain why the numerical minimization has worse performance for increasing $R e$, although it could be because the non-smoothness issue gets worse as $R e$ increases. The tendency of the eigenvalues to approach the imaginary axis at varying rates as $R e$ is increased may have something to do with it.

\section{Conclusion}

The aim of this paper was to find a method to disturb the laminar flow such that the disturbed state evolves and hits a given traveling wave. For the asymmetric traveling wave of Pringle and Kerswell (2007), we showed that certain linear combinations of rolls and the two unstable directions generate disturbances of the laminar solution that evolve and hit the traveling wave. Numerical minimization was used to find linear combinations that achieve that effect. As the numerical minimization comes closer and closer to finding disturbances that evolve and hit the traveling wave, the minimization problem becomes non-smooth.

It is reasonable to conjecture that this method of disturbing the laminar flow so that it evolves and hits a given traveling wave is applicable to other lower branch solutions of pipe flow and the channel flows. However, the computational effort will increase with the number of unstable directions. As indicated in the text, it may be possible to use analysis to find disturbances that work better than the unstable eigenvectors of the traveling wave.

It is yet unknown if spatially localized structures such as puffs in transitional pipe flow and turbulent spots in plane Couette flow correspond in their entirety to invariant solutions of the Navier-Stokes equation. The existence of such invariant solutions is a topic worthy of investigation. If such solutions are indeed found, the logic used to find 
disturbances that evolve and hit the asymmetric traveling wave will become applicable to transition in pipes of realistic lengths with consequences for experimental investigations. Acknowledgments. The authors thank J.F. Gibson and the referees for helpful discussions. The Center for Advanced Computing at the University of Michigan provided computing facilities. DV was partly supported by NSF grants DMS-0407110 and DMS0715510. PC was partly supported by NSF grant DMS-0807574.

\section{REFERENCES}

P. Cvitanović, R.L. Davidchack, and E. Siminos. State space geometry of a spatio-temporally chaotic Kuramoto-Sivashinsky flow. 2007. Available at arXiv:0709.2944.

A.G. Darbyshire and T. Mullin. Transition to turbulence in constant-mass-flux pipe flow. Journal of Fluid Mechanics, 289:83-114, 1995.

Y. Duguet, A.P. Willis, and R.R. Kerswell. Transition in pipe flow: the saddle structure on the boundary of turbulence. Journal of Fluid Mechanics, 613:255-274, 2008. Available at arXiv:0711.2175.

B. Eckhardt and T. Schneider. How does flow in a pipe become turbulent? European Physical Journal B, 64:457-462, 2008.

H. Faisst and B. Eckhardt. Sensitive dependence on initial conditions in transition to turbulence in pipe flow. Journal of Fluid Mechanics, 504:343-352, 2004.

H. Faisst and B. Eckhardt. Traveling waves in pipe flow. Physical Review Letters, 91:224502, 2003.

J.F. Gibson, J. Halcrow, and P. Cvitanović. Visualizing the geometry of state space in plane Couette flow. Journal of Fluid Mechanics, 611:107-130, 2008. Available at arXiv:0705.3957.

R. Gilmore and C. Letellier. The Symmetry of Chaos. Oxford University Press, Oxford, 2007.

J. Halcrow, J. F. Gibson, P. Cvitanović, and D. Viswanath. Heteroclinic connections in plane Couette flow. Journal of Fluid Mechanics, 2009. In press.

B. Hof, A. Juel, and T. Mullin. Scaling of the turbulence transition threshold in a pipe. Physical Review Letters, 91:244502, 2003.

B. Hof, C.W.H. van Doorne, et al. Experimental observation of nonlinear traveling waves in turbulent pipe flows. Science, 305:1594-1598, 2004.

T. Itano and S. Toh. The dynamics of bursting process in wall turbulence. Journal of the Physical Society of Japan, 70:701-714, 2001.

G. Kawahara. Laminarization of minimal plane Couette flow: going beyond the basin of attraction of turbulence. Physics of Fluids, 17:041702, 2005.

R.R. Kerswell. Recent progress in understanding the transition to turbulence in a pipe. Nonlinearity, 18:R17-R44, 2005.

R.R. Kerswell and O.R. Tutty. Recurrence of travelling waves in transitional pipe flow. Journal of Fluid Mechanics, 584:69-102, 2007.

G. Kreiss, A. Lundbladh, and D.S. Henningson. Bounds for threshold amplitudes in subcritical shear flows. Journal of Fluid Mechanics, 270:175-198, 1994.

M.T. Landahl. A note on an algebraic instablity of inviscid parallel shear flows. J. Fluid Mech., 98:243-251, 1980.

F. Mellibovsky and A. Meseguer. The role of streamwise perturbations in pipe flow transition. Physics of Fluids, 18:074104, 2006.

F. Mellibovsky and A. Meseguer. Pipe flow transition threshold following localized impulsive perturbations. Physics of Fluids, 19:044102, 2007.

M. Nagata. Three dimensional finite amplitude solutions in plane Couette flow: bifurcation from infinity. Journal of Fluid Mechanics, 217:519-527, 1990.

R. Narasimha. The utility and drawbacks of traditional approaches. In J. Lumley, editor, Whither Turbulence? Turbulence at the Cross-Road, pages 13-48. Springer-Verlag, Berlin, 1989.

P.L. O'Sullivan and K.S. Breuer. Transient growth in circular pipe flow ii. Physics of Fluids, 6: 3652-3664, 1994.

J. Peixinho and T. Mullin. Finite-amplitude thresholds for transition in pipe flow. Journal of Fluid Mechanics, 582:169-178, 2007. 
C.C.T. Pringle and R.R. Kerswell. Asymmetric, helical, and mirror-symmetric traveling waves in pipe flow. Physical Review Letters, 99:074502, 2007.

O. Reynolds. An experimental investigation of the circumstances which determine whether the motion of water shall be direct or sinuous, and of the law of resistance in parallel channels. Philosophical Transactions of the Royal Society of London, 174:935-982, 1883.

S.K. Robinson. Coherent motions in the turbulent boundary layer. Annual Review of Fluid Mechanics, 23:601-639, 1991.

A. Schmiegel and B. Eckhardt. Fractal stability border in plane Couette flow. Physical Review Letters, 79:5250, 1997.

T.M. Schneider, B. Eckhardt, and J. Vollmer. Statistical analysis of coherent structures in transitional pipe flow. Physical Review E, 75:066313, 2007a.

T.M. Schneider, B. Eckhardt, and J.A. Yorke. Turbulence transition and edge of chaos in pipe flow. Physical Review Letters, 99:034502, 2007b.

T.M. Schneider, J.F. Gibson, M. Lagha, F. De Lillo, and B. Eckhardt. Laminar-turbulent boundary in plane Couette flow. Physical Review E, 78:037301, 2008. Available at arXiv:0805.1015.

S. Toh and T. Itano. A periodic-like solution in channel flow. Journal of Fluid Mechanics, 481: 67-76, 2003.

L.N. Trefethen. Spectral Methods in Matlab. SIAM, Philadelphia, 2000.

D. Viswanath. Recurrent motions within plane Couette turbulence. Journal of Fluid Mechanics, 580:339-358, 2007.

D. Viswanath. The dynamics of transition to turbulence in plane Couette flow. In Mathematics and Computation, a Contemporary View. The Abel Symposium 2006, volume 3 of Abel Symposia. Springer-Verlag, Berlin, 2008a. Available at arXiv:0701337.

D. Viswanath. The critical layer in pipe flow at high Re. Philosophical Transactions of the Royal Society A, 2008b. To appear.

F. Waleffe. Three-dimensional coherent states in plane shear flows. Physical Review Letters, 81: 4140-4143, 1998.

J. Wang, J.F. Gibson, and F Waleffe. Lower branch coherent states in shear flows: transition and control. Physical Review Letters, 98:204501, 2007.

H. Wedin and R.R. Kerswell. Exact coherent structures in pipe flow: travelling wave solutions. Journal of Fluid Mechanics, 508:333-371, 2004.

A.P. Willis and R.R. Kerswell. Coherent structures in localised and global pipe turbulence. Physical Review Letters, 100:124501, 2008.

A.P. Willis and R.R. Kerswell. Turbulent dynamics of pipe flow captured in a reduced model: puff relaminarisation and localised 'edge' states. Journal of Fluid Mechanics, 2009. Available at arXiv:0712.2739.

A.P. Willis, J. Peixinho, R.R. Kerswell, and T. Mullin. Experimental and theoretical progress in pipe flow transition. Philosophical Transactions of the Royal Society A, 366:2671-2684, 2008.

I. Wygnanski, M. Sokolov, and D. Friedman. On transition in a pipe. Part 2. The equilibrium puff. Journal of Fluid Mechanics, 69:283-304, 1975.

I.J. Wygnanski and F.H. Champagne. On transition in a pipe. Part 1. The origin of puffs and slugs and the flow in a turbulent slug. Journal of Fluid Mechanics, 59:281-335, 1973. 